\title{
cmaJOPEN
}

\section{Health service use among Manitobans with alcohol use disorder: a population-based matched cohort study}

\author{
James M. Bolton MD, Christine Leong PharmD, Okechukwu Ekuma MSc, Heather J. Prior MSc, \\ Geoffrey Konrad MD, Jennifer Enns PhD, Deepa Singal PhD, Josh Nepon MD, Michael T. Paillé MSc, \\ Greg Finlayson PhD, Nathan C. Nickel PhD
}

\section{Abstract}

Background: Alcohol is the drug most commonly used by Canadians, with multiple impacts on health and health service use. We examined patterns of short- and long-term health service use among people with a diagnosis of alcohol use disorder.

Methods: In this retrospective matched cohort study, we used population-based administrative data from the province of Manitoba, Canada, to identify individuals aged 12 years or older with a first indication of alcohol use disorder (index date) in the period 1990 to 2015. We matched cases (those with diagnosis of alcohol use disorder) to controls (those without this diagnosis), at a 1:5 ratio, on the basis of age, sex, geographic region and income quintile at the index date. The outcome measures were inpatient hospital admission, outpatient physician visits, emergency department visits and use of prescription medications. We modelled crude rates using generalized estimating equations with either a negative binomial or a Poisson distribution

Results: We identified 53410 people with alcohol use disorder and 264857 matched controls. All outcomes occurred at a higher rate among people with the disorder than among controls. For example, during the year of diagnosis, the rate ratio for hospital admission was 4.0 (95\% confidence interval [Cl] 3.9-4.2) for women and $4.5(95 \% \mathrm{Cl} 4.4-4.7)$ for men. All rates of health service use peaked close to the index date, but remained significantly higher among people with alcohol use disorder than among controls for 20 years. Among people with alcohol use disorder, the most commonly filled prescriptions were for psycholeptics, whereas among controls, the most commonly filled prescriptions were for sex hormones (women) and antihypertensives (men).

Interpretation: Compared with controls, people with alcohol use disorder used significantly more health services from the time of diagnosis and over the next 20 years. This finding highlights the need for better detection and early intervention to reduce the need for acute and emergency care, as well as the need for improved management of alcohol use disorder over the longer term.

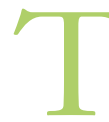

he high prevalence of alcohol consumption in Canada is a contributing risk factor for several chronic disease states. ${ }^{1-3}$ According to recent estimates, more than three-quarters of Canadians consume alcohol regularly, with almost 1 in 5 surpassing the level of consumption that puts their health at elevated chronic risk. ${ }^{4}$ Alcohol use disorder, a manifestation of at-risk alcohol consumption, is characterized by a pattern of alcohol drinking associated with substantial impairment or distress. ${ }^{5}$ For 2012, Statistics Canada reported that the annual prevalence of alcohol use disorder was about $4.7 \%$ for men and $1.7 \%$ for women. ${ }^{6}$ The total costs and harms attributable to alcohol use, including health-related costs, lost workplace productivity and criminal justice costs, are estimated to be nearly $\$ 15$ billion and are higher for alcohol than for any other substance measured. ${ }^{7}$ Many studies have linked alcohol use disorder to increased risk of chronic disease (including liver cirrhosis, cognitive impairment and cancer), impair- ment of interpersonal relationships and occupational functioning, injury, violence and suicide. ${ }^{8-11}$

Despite the high degree of medical and psychosocial comorbidity associated with alcohol use disorder, relatively little is known about patterns of health service use in the general population of people with the disorder. Previous studies have examined hospital stays among "risky drinkers" who did not have a diagnosis of alcohol use disorder, ${ }^{12}$ have reported cross-sectional rates of emergency department visits related to alcohol use in small samples of people with diagnosis of the

\section{Competing interests: None declared.}

This article has been peer reviewed.

Correspondence to: Nathan Nickel, Nathan_Nickel@ umanitoba.ca CMAJ Open 2020. DOI:10.9778/cmajo.20200124 
disorder ${ }^{13-16}$ and have used survey data to describe rates of health service use among people with alcohol use disorder in Canada ${ }^{17}$ and the United States. ${ }^{18}$ Understanding patterns of health service use among people with diagnosis of this disorder could help inform strategies for providing more appropriate and effective care for this population.

We examined the short- and long-term rates of hospital admission, length of hospital stay, physician visits, emergency department visits and use of prescription medications among people in Manitoba, Canada, with a diagnosis of alcohol use disorder. Given the disease burden associated with heavy alcohol consumption, we hypothesized that people with this diagnosis would have higher rates of health service use than matched controls without the diagnosis.

\section{Methods}

\section{Design and setting}

Using administrative data from the Manitoba Population Research Data Repository, housed at the Manitoba Centre for Health Policy, we conducted a retrospective, populationbased cohort study of Manitobans with an indication for alcohol use disorder. The repository contains more than 90 databases of information collected during administration of the health care system, the education system, social services and the justice system; it thus represents an information-rich resource for examining the health and well-being of the population of Manitoba.

\section{Data sources}

We accessed the following specific databases for this study: Medical Services Data (diagnoses for mental and physical disorders from outpatient physician contacts), Hospital Abstracts Data (inpatient hospitalization, in-hospital diagnoses for mental and physical disorders), the Manitoba Health Insurance Registry (age, sex, region of residence), Canada Census data (average household income to generate income quintiles) and Drug Program Information Network (prescription drug dispensations). The repository data can be linked by way of encrypted identification numbers and have undergone validity checks showing their excellent linkage accuracy. ${ }^{19-21}$

\section{Study population}

To create the exposure group (cases), we identified all Manitobans aged 12 years or older with first diagnosis of an alcohol use disorder between Apr. 1, 1990, and Mar. 31, 2015. We considered the date of first diagnosis as the "index date" for purposes of matching and subsequent analyses. We used a 7-year washout period (Apr. 1, 1983, to Mar. 31, 1990) to ascertain the first diagnosis (whereby cases could not have any other documented diagnoses of alcohol use disorder in the 7 years leading up to the first diagnosis).

We defined alcohol use disorder according to codes from the clinical modification of the International Classification of Diseases, 9th Revision (ICD-9-CM) and the Canadian version of the International Statistical Classification of Diseases and Related Health Problems, 10th Revision (ICD-10-CA). A person was coded as having alcohol use disorder if a physical or mental alcohol-related ICD-9-CM or ICD-10-CA code was recorded for an outpatient physician visit or inpatient admission during the study period, or if at least 1 prescription was dispensed for medications used to treat alcohol use disorders (see Appendix 1, Table S1, available at www.cmaj open.ca/content/8/4/E762/suppl/DC1, for the full list of ICD-9-CM and ICD-10-CA codes and prescription medications used in our definition of alcohol use disorder).

To create the control group, we matched individuals from the general Manitoba population with at least 5 years of continuous health coverage before the index date to cases, at a 5:1 ratio. We excluded people from the control group if they met the criteria for alcohol use disorder during the study or washout period, if they had ever had diagnosis of a substance use disorder of any kind, if they had ever been accused of driving while intoxicated, if they had ever reported that they drank during pregnancy or if they were mothers of children with fetal alcohol spectrum disorder (see Appendix 1, Table S2, for more details).

We matched individuals eligible for the control group to cases on the basis of sex, date of birth $( \pm 2.5 \mathrm{yr})$, geographic region (on the basis of 3-digit postal code) and income quintile at the index date. We followed the cases and controls from 5 years before the index date until the end of the study period, death or loss of health insurance coverage (i.e., moved out of the province).

\section{Main outcomes}

The main health service use outcomes were inpatient hospitalization, outpatient physician visits, emergency department visits and prescription medication use (see Appendix 1, Box S1 for detailed definitions). Specific hospitalization outcomes were annual rates of hospital admission per 1000 person-years, annual number of days spent in hospital per 100 person-years and the 10 most common diagnostic reasons for hospital admission. We counted multiple admissions for the same individual during the study period as separate events.

We estimated the rate of outpatient visits to physicians (general and specialist practitioners) per person-year. We captured Winnipeg residents' emergency department visits for the 6 Winnipeg hospitals as rates per 100 person-years. Measures of prescription medication use were both the number of different types of medications dispensed and the 10 most commonly dispensed medications by Anatomic Therapeutic Chemical classification category.

\section{Predictor variables}

To adjust for differences between the cases and controls, we included age at time of diagnosis and calendar year in the statistical models. We also included postal code of residence (which accounts for both region of residence and income, because an individual might have changed residence during the course of the study, after being matched) and mental disorders (defined using diagnostic codes, as described in Appendix 1, Table S1) as time-varying characteristics in the models. 


\section{Other measures}

We assigned Johns Hopkins Resource Utilization Bands, based on the Johns Hopkins Adjusted Clinical Group CaseMix System (version 10), ${ }^{22}$ for the study cohort. This measure is a simplified ranking system of overall morbidity level (not type of illness), such that individuals expected to use the same level of resources are grouped together, even if they have very different illnesses with different epidemiologic patterns. ${ }^{22}$ Individuals were assigned a resource utilization band from 0 (non-user) to 5 (very high morbidity).

\section{Statistical analysis}

We assessed outcomes from 5 years before to 20 years after the index date or loss to follow-up. We also investigated these patterns by age group among women and men. We modelled crude rates using generalized estimating equations with either a negative binomial or Poisson distribution and a $\log$ offset of person-years to account for different observation times, generating rate ratios adjusted for mental health disorders, age at index diagnosis, region of residence and income level. We calculated adjusted rates using multivariable generalized estimating equations that included the predictor variables listed above. We selected the equations to account for the hierarchical nature of our analyses - within-person clustering, matching and within-geography clustering. We ranked the most common diagnostic reasons for hospital admission and the most commonly dispensed prescription medications by frequency and presented them as proportions of the 10 most common categories in the year before, the year after and the fifth year after the index date. We conducted all analyses using SAS version 9.4 software (SAS Institute Inc.).

\section{Ethics approval}

The study was approved by the University of Manitoba's Human Research Ethics Board.

\section{Results}

The characteristics of people with alcohol use disorder $(n=$ $53410)$ and their matched controls $(n=264857)$ are presented in Table 1. The groups with the highest frequency of new diagnosis were young adults (18-24 yr) and both urbanand rural-dwelling individuals with low socioeconomic status. We observed a bimodal pattern of disease comorbidity among people with alcohol use disorder: most fell into either the lowest health resource use category (resource utilization band 0) or the middle category (resource utilization band 3). Rates of comorbid mood and anxiety disorders were high for both men and women with alcohol use disorder.

Rate ratios describing the association between diagnosis of alcohol use disorder and each of the main outcomes are presented in Table 2. All health service use outcomes that we measured were significantly higher for people with alcohol use disorder during the year of first diagnosis than for the controls. The rate of hospital admission was at least 4 times higher among both women and men with alcohol use disorder, and women spent almost 8 times and men spent 9 times longer in hospital. People with alcohol use disorder visited the emergency department 3 times more often than controls during the year after their first diagnosis.

Figure 1 shows the number of days that cases and controls spent in hospital from 5 years before the index date to 20 years after the index date. Among both women and men, we observed that annual inpatient hospital days among people with alcohol use disorder increased sharply in the years before first diagnosis, peaked during the year after diagnosis and then decreased over the next 20 years, but still remained higher than among controls (and higher than the prediagnosis rate among cases). We saw a similar pattern for outpatient physician visits and emergency department visits (Appendix 1, Figure S1 and Figure S2, respectively), although the peak in emergency department visits occurred in the year preceding diagnosis for both women and men. In our analyses of these patterns by age group among women and men, we found no substantive differences in these outcomes among any of the age groups and the overall cohort for either sex (data not shown).

Figure 2 shows the 10 most common reasons for hospital admission by ICD-10-CA chapter among cases and controls. Women and men with alcohol use disorder were most likely to be admitted for mental health and behavioural disorders in both the year of first diagnosis and the fifth year after the index date. Injury, poisoning and other consequences of external causes (e.g., trauma, fractures, burns, frostbite and poisoning by medications and illicit drugs not classified as intentional self-harm) were common causes of hospital admission for women and men with alcohol use disorder across all time points. Women in the control group were most often admitted for obstetric reasons, digestive diseases and circulatory diseases, whereas men in the control group were most commonly admitted for circulatory and digestive diseases.

Figure 3 presents the 10 most commonly dispensed prescription medications (by Anatomic Therapeutic Chemical classification category) among cases and controls. There were stark contrasts between the types of drugs dispensed to those with and without alcohol use disorder. For women with alcohol use disorder, the most commonly dispensed medications were psycholeptics (e.g., anxiolytics, antipsychotics, sedativehypnotics) and psychoanaleptics (e.g., antidepressants, psychostimulants) during all 3 time periods measured. For women without alcohol use disorder, sex hormones (e.g., contraceptives) were more commonly dispensed. For men with alcohol use disorder, psycholeptics were most commonly prescribed during all 3 time periods, followed by either psychoanaleptics or analgesics. For men without alcohol use disorder, agents acting on the renin-angiotensin system were most often prescribed, followed by diabetes medications and lipid-lowering agents.

\section{Interpretation}

In this longitudinal population-based cohort study, Manitobans with a diagnosis of alcohol use disorder had higher rates of health service use (including a fourfold higher rate of 


\begin{tabular}{|c|c|c|c|c|c|c|}
\hline \multirow[b]{3}{*}{ Characteristic } & \multicolumn{6}{|c|}{ Sex; study group; ${ }^{*}$ no. (\%) of participants $†$} \\
\hline & \multicolumn{3}{|c|}{ Women } & \multicolumn{3}{|c|}{ Men } \\
\hline & $\begin{array}{c}\text { Manitoba } \\
n=560102\end{array}$ & $\begin{array}{c}\text { Cases } \\
n=19017\end{array}$ & $\begin{array}{c}\text { Controls } \\
n=94566\end{array}$ & $\begin{array}{c}\text { Manitoba } \\
n=544672\end{array}$ & $\begin{array}{c}\text { Cases } \\
n=34393\end{array}$ & $\begin{array}{c}\text { Controls } \\
n=170291\end{array}$ \\
\hline $\begin{array}{l}\text { Age at diagnosis, yr, } \\
\text { mean }(95 \% \mathrm{Cl})\end{array}$ & $44.9(44.8-44.9)$ & $38.3(38.1-38.6)$ & $38.3(38.2-38.4)$ & $43.1(43.0-43.1)$ & $42.2(42.0-42.4)$ & $42.2(42.1-42.3)$ \\
\hline Age group, yr & NA & & & NA & & \\
\hline $12-17$ & & $2184(11.5)$ & 11030 (11.7) & & $1915(5.6)$ & 11097 (6.5) \\
\hline $18-24$ & & 3687 (19.4) & $18232(19.3)$ & & $5791(16.8)$ & 27537 (16.2) \\
\hline $25-29$ & & $2086(11.0)$ & $10232(10.8)$ & & $3276(9.5)$ & $15517(9.1)$ \\
\hline $30-34$ & & $1876(9.9)$ & $9346(9.9)$ & & $3202(9.3)$ & $15465(9.1)$ \\
\hline $35-39$ & & $1549(8.1)$ & $7704(8.2)$ & & $2970(8.6)$ & $14668(8.6)$ \\
\hline $40-44$ & & $1475(7.8)$ & $7356(7.8)$ & & $2792(8.1)$ & $14034(8.2)$ \\
\hline $45-49$ & & $1231(6.5)$ & $6133(6.5)$ & & $2678(7.8)$ & $13226(7.8)$ \\
\hline $50-54$ & & $972(5.1)$ & $4870(5.2)$ & & $2370(6.9)$ & $12002(7.0)$ \\
\hline $55-59$ & & $775(4.1)$ & $3852(4.1)$ & & $2183(6.3)$ & $10962(6.4)$ \\
\hline $60-64$ & & $684(3.6)$ & $3431(3.6)$ & & $1869(5.4)$ & $9316(5.5)$ \\
\hline $65-69$ & & $654(3.4)$ & $3232(3.4)$ & & $1792(5.2)$ & 8965 (5.3) \\
\hline $70-74$ & & $560(2.9)$ & $2789(3.0)$ & & $1458(4.2)$ & $7182(4.2)$ \\
\hline $75-79$ & & $548(2.9)$ & $2735(2.9)$ & & $1091(3.2)$ & $5412(3.2)$ \\
\hline $80-84$ & & $383(2.0)$ & $1889(2.0)$ & & $631(1.8)$ & $3103(1.8)$ \\
\hline $85-89$ & & 247 (1.3) & $1224(1.3)$ & & $294(0.9)$ & $1429(0.8)$ \\
\hline$\geq 90$ & & $106(0.6)$ & $511(0.5)$ & & $81(0.2)$ & $376(0.2)$ \\
\hline Urban residence & $353506(63.1)$ & $10527(55.4)$ & $52477(55.5)$ & 336087 (61.7) & $18940(55.1)$ & 94347 (55.4) \\
\hline \multicolumn{7}{|l|}{ Income quintile } \\
\hline Urban 1 (lowest) & $68287(12.2)$ & $4326(22.7)$ & 21607 (22.8) & 64495 (11.8) & 6727 (19.6) & 33586 (19.7) \\
\hline Urban 2 & 70370 (12.6) & $2131(11.2)$ & 10634 (11.2) & $66222(12.2)$ & 4109 (11.9) & $20519(12.0)$ \\
\hline Urban 3 & $69913(12.5)$ & $1661(8.7)$ & $8290(8.8)$ & $66089(12.1)$ & $3230(9.4)$ & $16113(9.5)$ \\
\hline Urban 4 & 69155 (12.3) & $1167(6.1)$ & $5813(6.1)$ & $67598(12.4)$ & $2541(7.4)$ & $12654(7.4)$ \\
\hline Urban 5 (highest) & 70751 (12.6) & $955(5.0)$ & $4774(5.0)$ & $68231(12.5)$ & $1958(5.7)$ & $9777(5.7)$ \\
\hline Rural 1 (lowest) & $38419(6.9)$ & 3096 (16.3) & $15343(16.2)$ & $38845(7.1)$ & $4976(14.5)$ & $24248(14.2)$ \\
\hline Rural 2 & $41532(7.4)$ & $1879(9.9)$ & 9337 (9.9) & $41626(7.6)$ & $3244(9.4)$ & $15925(9.4)$ \\
\hline Rural 3 & $42333(7.6)$ & $1193(6.3)$ & $5912(6.3)$ & $42326(7.8)$ & $2543(7.4)$ & $12641(7.4)$ \\
\hline Rural 4 & $41124(7.3)$ & $1216(6.4)$ & $6063(6.4)$ & $41872(7.7)$ & $2383(6.9)$ & $11794(6.9)$ \\
\hline Rural 5 (highest) & $41876(7.5)$ & $1053(5.5)$ & $5251(5.6)$ & $42813(7.9)$ & $2230(6.5)$ & $11087(6.5)$ \\
\hline Unknown & $6342(1.1)$ & $340(1.8)$ & $1542(1.6)$ & $4555(0.8)$ & $452(1.3)$ & $1947(1.1)$ \\
\hline \multicolumn{7}{|l|}{ RUB } \\
\hline 0 (non-users) & $89515(16.0)$ & 6209 (32.6) & 31197 (33.0) & $156053(28.7)$ & $15728(45.7)$ & $77863(45.7)$ \\
\hline 1 (lowest morbidity) & $54857(9.8)$ & $687(3.6)$ & $6710(7.1)$ & $55758(10.2)$ & $1322(3.8)$ & $10619(6.2)$ \\
\hline 2 & $139029(24.8)$ & 2707 (14.2) & $19234(20.3)$ & $133932(24.6)$ & $4738(13.8)$ & $29650(17.4)$ \\
\hline 3 & $248581(44.4)$ & 7757 (40.8) & $33718(35.7)$ & $175721(32.3)$ & $10361(30.1)$ & $45652(26.8)$ \\
\hline 4 & $23898(4.3)$ & $1416(7.4)$ & $3191(3.4)$ & $19391(3.6)$ & $1872(5.4)$ & 5467 (3.2) \\
\hline 5 (highest morbidity) & $4222(0.8)$ & $241(1.3)$ & $516(0.5)$ & $3817(0.7)$ & $372(1.1)$ & $1040(0.6)$ \\
\hline \multicolumn{7}{|l|}{ Mental disorder } \\
\hline Mood or anxiety disorder & $155809(27.8)$ & $9809(51.6)$ & $19428(20.5)$ & $85360(15.7)$ & 10724 (31.2) & $18608(10.9)$ \\
\hline Personality disorder & $18009(3.2)$ & $1226(6.4)$ & $723(0.8)$ & $13998(2.6)$ & $1330(3.9)$ & $1035(0.6)$ \\
\hline Psychosis & $8633(1.5)$ & $1269(6.7)$ & $1541(1.6)$ & $8394(1.5)$ & $2218(6.4)$ & $2678(1.6)$ \\
\hline
\end{tabular}




\begin{tabular}{|c|c|c|}
\hline \multirow[b]{2}{*}{ Variable } & \multicolumn{2}{|c|}{ Sex; adjusted rate ratio $(95 \% \mathrm{Cl})$} \\
\hline & Women & Men \\
\hline Hospital admissions & $4.0(3.9-4.2)$ & $4.5(4.4-4.7)$ \\
\hline Outpatient physician visits & $1.7(1.7-1.8)$ & $2.0(1.9-2.0)$ \\
\hline No. of medications prescribed & $1.8(1.7-1.8)$ & $1.9(1.8-1.9)$ \\
\hline Emergency department visits $†$ & $3.8(3.4-4.3)$ & $3.6(3.2-3.9)$ \\
\hline Days in hospital & $7.9(7.1-8.8)$ & $9.2(8.4-10.1)$ \\
\hline \multicolumn{3}{|c|}{$\begin{array}{l}\text { Note: } \mathrm{Cl}=\text { confidence interval. } \\
\text { *Adjusted for mental health disorders, age at index diagnosis, region of } \\
\text { residence and income level. } \\
\text { †Visits included only those made by Winnipeg residents to any of the } 6 \text { Winnipeg } \\
\text { emergency departments (women, } n=7548 \text {; men, } n=12833 \text { ). }\end{array}$} \\
\hline
\end{tabular}

hospital admission and a threefold higher rate of emergency department visits) than a cohort of matched controls. Health service use was highest in the years before and after the first diagnosis of alcohol use disorder, and was sustained at a higher rate over the subsequent 20 years. The study also showed differences in the patterns of medication use between people with alcohol use disorder and controls, with higher use of psychotropic medications among those with the disorder. Together, these findings underscore a concerning profile of substantial morbidity among people with alcohol use disorder, highlighting a considerable burden on multiple areas of the health system. This finding has important implications for health service and social services planning, particularly with regard to the need for coordination of treatment among providers and across services.

The study showed that people with alcohol use disorder had significantly elevated health service use. In the years leading up to their diagnosis, they had a pattern of health care use distinct from their counterparts without alcohol use disorder, having more frequent hospital admission and admission for different reasons. In the year before diagnosis of alcohol use disorder, men were most frequently admitted for injuries and poisonings and had a 6 times higher rate of emergency department visits, which suggests a chaotic and behaviourally high-risk lifestyle, perhaps related to the social determinants of health associated with adversity. Women similarly experienced heightened health service use in the year before (emergency department visits, outpatient visits) and after (hospital admission) diagnosis of alcohol use disorder. These findings align with a recent report from Ontario highlighting a 4.4 times higher rate of alcohol-related emergency department visits, over and above the increase in rate for all emergency department visits combined from 2003 to $2016 .^{23}$

We also found it notable that among both men and women, the 18- to 24-year-old group made up the largest proportion of those with alcohol use disorder $(16.8 \%$ for men and $19.4 \%$ for women). The high rates of alcohol use disorder in this age group, among young women in particular, are concerning. The Canadian Centre on Substance Abuse and
Addiction has reported that the 2015 past-year prevalence of alcohol consumption among women aged 18 to 24 years was $81.4 \%$, higher than in any other age group. ${ }^{24}$ Women of child-bearing age have a higher risk of consuming alcohol during pregnancy than women in other age groups, and younger women may be particularly vulnerable to experiencing aggression or gender-related violence while drinking. ${ }^{25-27}$ As well, our study shows that women with alcohol use disorder experience substantially higher rates of mood and anxiety disorders than men with this disorder. Consistent assessments of alcohol intake at emergency department admissions and appropriate intervention following emergency visits could play an important role in preventing problem drinking behaviours in young adults and the eventual development of alcohol use disorder and other mental illness.

In our study, a diagnosis of alcohol use disorder coincided with substantially higher service use across multiple areas of the health care system, possibly because many people with this disorder do not perceive a need for treatment ${ }^{28}$ and thus may present for care only when their health conditions have become acute. The "spike" in health service use at or just before diagnosis shown in Figure 1 (and in Appendix 1, Figure S3 and Figure S4) may have been the result of an alcoholrelated "health crisis" that brought individuals to health care settings where their alcohol use disorder could be identified. The high prevalence of hospital visits for mental or behavioural disorders and for injuries and poisoning before and after diagnosis of alcohol use disorder (shown in Figure 2) suggests that substantial mental illness comorbidities were present among many individuals with alcohol use disorder, and may help to explain why the use of all types of health services that we measured remained higher for the 2 decades after diagnosis.

The higher rates of inpatient hospital admission that we observed in this study are consistent with a previous longitudinal study in Spain, in which hospital admission rates were lowest among alcohol abstainers compared with those who consumed moderate to heavy levels of alcohol at 20-year follow-up. ${ }^{29}$ In that study, heavy alcohol drinking was also associated with higher rates of hospital days than abstaining or and drinking moderately.

Our findings on the types of prescription medications used by people with alcohol use disorder likely reflect the high comorbidity between this and other mental disorders ${ }^{30}$ and also raise the possibility that prescription drugs obtained by this population, such as benzodiazepines, may be misused or diverted, contributing to concern about how the general health of people with alcohol use disorder is managed. It is also possible, given the overriding importance of psycholeptic and psychoanaleptic medications in treating this population, that management of other nonpsychiatric health problems is delayed. Analgesics were also commonly prescribed for people with alcohol use disorder (second or third most common category in Figure 3), which suggests that an underlying reason for excessive alcohol use may be for pain management. We observed that age-matched women with alcohol use disorder filled prescriptions for contraceptives or other sex hormones 

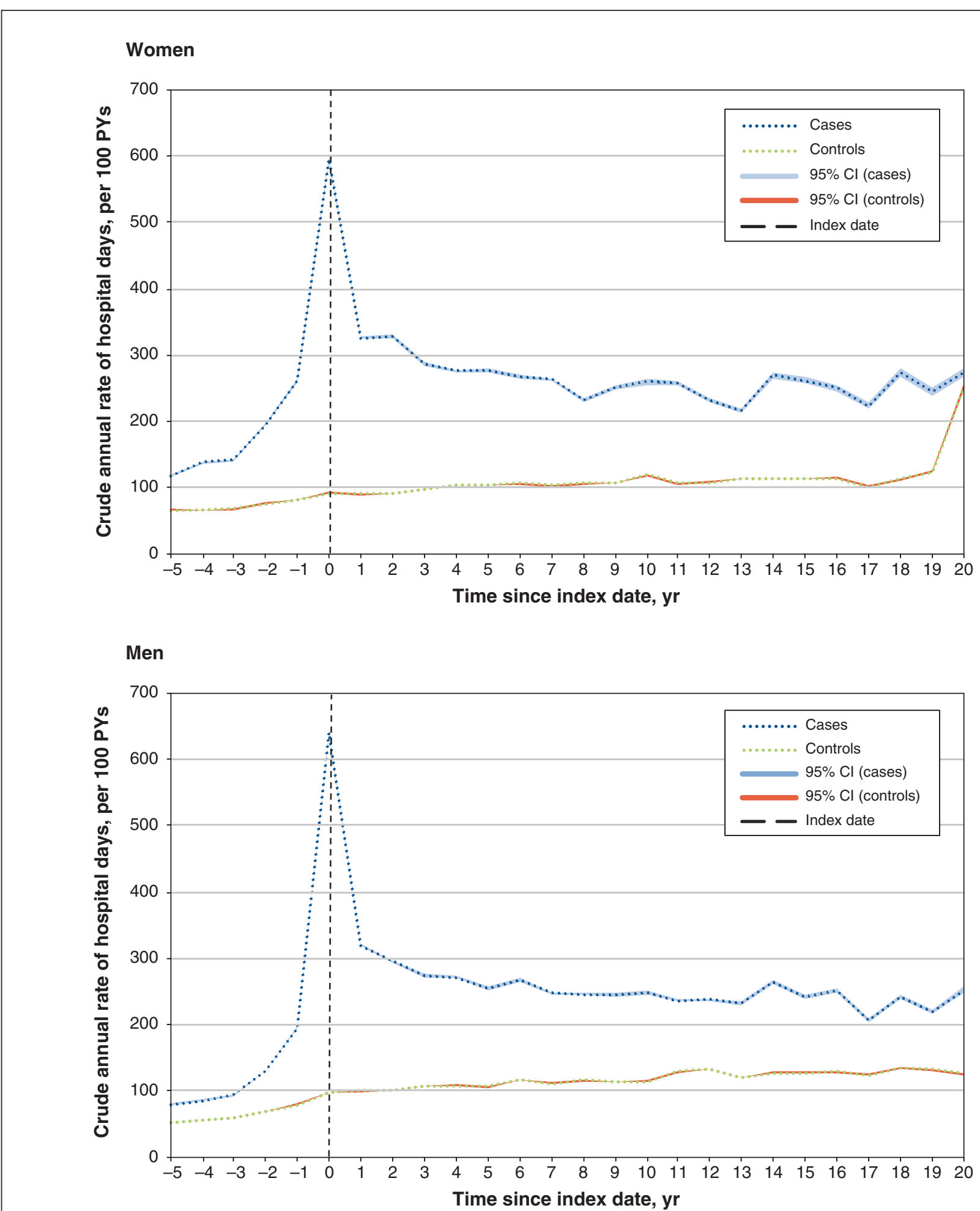

Figure 1: Crude annual rates of hospital days for women (top) and men (bottom) with and without alcohol use disorder (cases and controls, respectively), before and after the index date, per 100 person-years (PYs), where the index date was the date of first diagnosis of alcohol use disorder. Note: $\mathrm{Cl}=$ confidence interval.

much less frequently than women without the disorder. This observation, coupled with known associations between alcohol use disorder, impulsivity and risky sexual behaviour, ${ }^{31,32}$ could lead to a series of adverse downstream effects. As well, men with alcohol use disorder filled prescriptions for diabetes medications and renin-angiotensin inhibitors far less 


\section{Women}
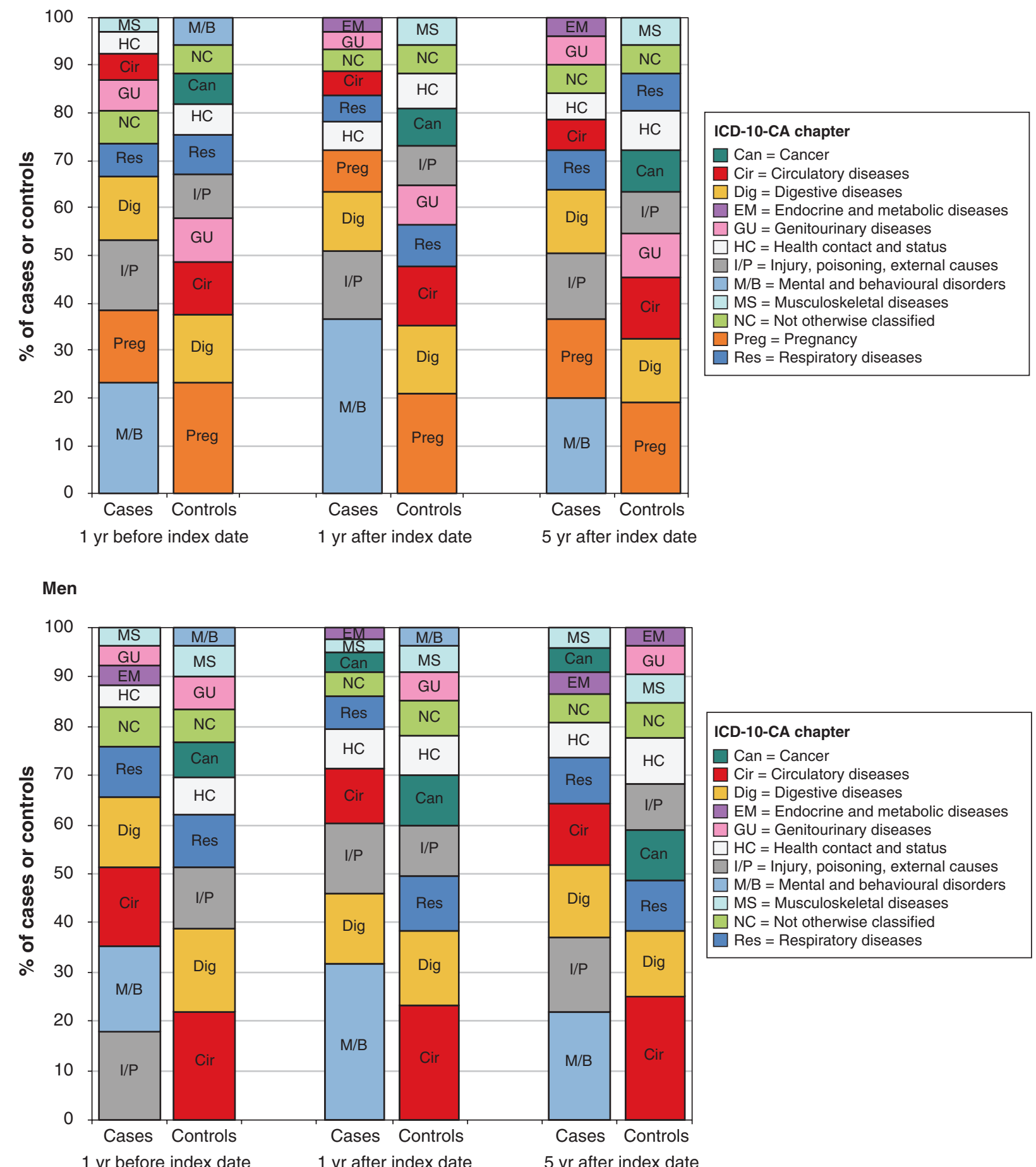

Figure 2: Ten most common causes of admission to hospital, according to chapters of the Canadian version of the International Statistical Classification of Diseases and Related Health Problems, 10th Revision (ICD-10-CA) for women (top) and men (bottom) with and without alcohol use disorder (cases and controls, respectively) in the year before, the year after and 5 years after the index date. Hospital admissions included obstetric admissions, and these data cover admissions of Manitobans to hospitals in any Canadian province.

frequently than did controls, and lipid-lowering agents were not among their 10 most frequently dispensed agents. Together, these findings suggest that people with alcohol use disorder experience poor pharmacologic management of common health conditions, which likely contributes to their higher rates of physical disorder morbidity. 


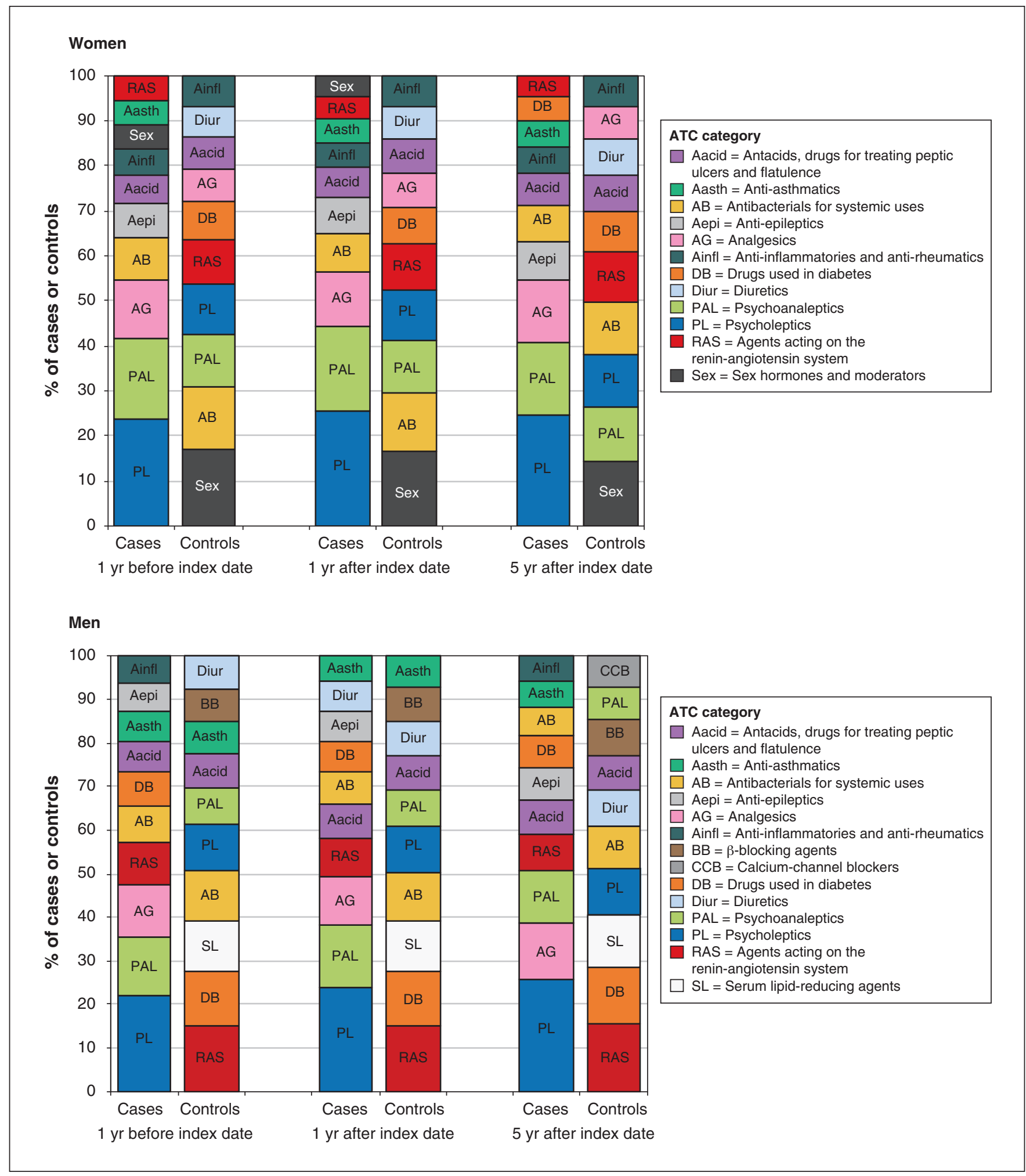

Figure 3: Ten most commonly dispensed prescription medications by Anatomic Therapeutic Chemical (ATC) classification category for women (top) and men (bottom) with and without alcohol use disorder (cases and controls, respectively) in the year before, the year after and 5 years after index date. Nonprescription medications and medications from hospital-based pharmacies were excluded.

The study findings highlight important opportunities for earlier identification of high-risk drinking patterns. Primary care practitioners and specialists in other disciplines should be aware of alcohol disorder screening guidelines ${ }^{33}$ and should implement them consistently in their practices. Brief interventions for at-risk drinking and alcohol use disorder in primary 
care settings have been shown to reduce heavy drinking and its associated adverse outcomes. ${ }^{34-36}$ Adopting upstream approaches to intervene before alcohol use disorder fully develops will be important for mitigating the short- and longterm harm experienced by heavy users of alcohol.

\section{Limitations}

Studies of this type have important limitations relating to the use of administrative databases. Because there was no specific code for alcohol use disorder, we developed a definition using ICD-9-CM and ICD-10-CA codes for alcohol-related physical and mental health diagnoses and receipt of prescription drugs used to treat alcohol use disorder. This approach has been validated ${ }^{37}$ but may have resulted in inclusion of only those cases severe enough for development of an alcoholrelated complication, whereas people with subthreshold but excessive drinking patterns might have been missed. Previous studies have reported that as many as $80.4 \%$ of people with alcohol use disorder do not seek treatment, ${ }^{38}$ and it is therefore difficult to capture people in the early stages of the disorder using administrative claims data.

Nonetheless, the findings from our study provide important insights into this high-risk population, and we can be certain that our study population had patterns of alcohol use resulting in harm. Although we were able to follow the course of health service use among individuals with alcohol use disorder, it was difficult to determine the impact over time of changing drinking patterns (given the relapsing and remitting nature of the disorder) or the length of abstinence after receipt of an alcohol-related diagnosis.

The study examined alcohol use disorder in the general population of Manitoba, but we did not have permission to disaggregate the study population by First Nations (or other Indigenous) identity; therefore, the study lacks the richness that such an analysis would have added. There may be a small amount of residual unmeasured confounding from not accounting directly for First Nations identity (or for variables such as socioeconomic status); however, we matched cases and controls on income quintiles and region of residence, based on 3-digit postal codes, thus minimizing the geographic distance between their places of residence, which mitigates some of the risk for confounding by ensuring that cases were matched with controls living in the same community.

Data concerning emergency department visits were available only for Winnipeg residents visiting Winnipeg hospitals, and we could not capture visits to emergency departments outside Winnipeg. This limits the generalizability of these findings to some degree, although we believe the overall effect on the findings to be minimal.

This observational study did not allow us to examine causation between alcohol use disorder and health service use outcomes. We also recognize that important questions remain unanswered, including the specific reasons for higher health service use by individuals with this disorder (e.g., health impairment as a direct result of alcohol use disorder v. more general health problems like impaired immunity) and details about the duration and severity of the disorder, as well as any changes to health service use among individuals who are in remission. Future studies will delve into these deeper queries to address the knowledge gaps more fully.

\section{Conclusion}

Men and women with alcohol use disorder used health services at a higher rate than controls, in both the 5-year and 20-year periods after diagnosis. A spike in service use was seen in the years before and after diagnosis. These findings point to opportunities for improved detection and intervention early in alcohol use disorder, to reduce the need for acute or emergency care, and highlight a need for improved management of alcohol use disorder over the longer term.

\section{References}

1. Global status report on alcohol and health 2018. Geneva: World Health Organization; 2018. Available: https://apps.who.int/iris/bitstream/handle/10665/ 274603/9789241565639-eng.pdf?ua =1 (accessed 2020 Oct. 10).

2. Rehm J, Baliunas D, Borges G, et al. The relation between different dimensions of alcohol consumption and burden of disease: an overview. Addiction 2010;105:817-43.

3. Dawson DA, Li T, Grant B. A prospective study of risk drinking: At risk for what? Drug Alcohol Depend 2008;95:62-72.

4. Canadian alcohol and drug use monitoring survey: summary of results for 2012. Ottawa: Health Canada; 2012. Available: https://www.canada.ca/en/health-canada/ services/health-concerns/drug-prevention-treatment/drug-alcohol-use-statistics/ canadian-alcohol-drug-use-monitoring-survey-summary-results-2012.html (accessed 2017 Sept. 2)

5. Alcohol-related disorders. In: Diagnostic and statistical manual of mental disorders. 5th ed. Arlington (VA): American Psychiatric Association Publishing; 2013. Available: https://dsm-psychiatryonline-org.uml.idm.oclc.org/doi/full/10.1176/ appi.books.9780890425596.dsm16 (accessed 2020 Oct. 10). Login required to access content.

6. Chart 2: Rates of substance use disorders, 12-month, by sex, Canada, housebold population 15 and older, 2012. Ottawa: Statistics Canada; 2012. Cat no 82-624-X. Available: https://www150.statcan.gc.ca/n1/pub/82-624-x/2013001article/c-g/11855-c-g-02 -eng.htm (accessed 2019 Dec. 11).

7. Canadian Substance Use Costs and Harms Scientific Working Group. Canadian substance use costs and harms (2007-2014). Ottawa: Canadian Centre on Subtance Use and Addiction; 2018. Available: https://www.ccsa.ca/canadian -substance-use-costs-and-harms (accessed 2020 Oct. 10).

8. Rehm J, Mathers C, Popova S, et al. Global burden of disease and injury and economic costs attributable to alcohol use and alcohol-use disorders. Lancet 2009;373:2223-33.

9. Bouchery EE, Harwood H, Sacks J, et al. Economic costs of excessive alcohol consumption in the U.S., 2006. Am 7 Prev Med 2011;41:516-24.

10. Bagnardi $V$, Rota $M$, Botteri $E$, et al. Light alcohol drinking and cancer: a meta-analysis. Ann Oncol 2013;24:301-8.

11. Hill SY, Steinhauer SR, Locke-Wellman J, et al. Childhood risk factors for young adult substance dependence outcome in offspring from multiplex alcohol dependence families: a prospective study. Biol Psychiatry 2009;66:750-7.

12. Miquel L, Manthey J, Rehm J, et al. Risky alcohol use: the impact on health service use. Eur Addict Res 2018;24:234-44.

13. McNicholl B, Goggin D, O'Donovan D. Alcohol-related presentations to emergency departments in Ireland: a descriptive prevalence study. BM7 Open 2018;8:e021932.

14. Egerton-Warburton D, Gosbell A, Moore K, et al. Alcohol-related harm in emergency departments: a prospective, multicenter study. Addiction 2018;113:623-32.

15. Egerton-Warburton D, Gosbell A, Wadsworth A, et al. Survey of alcohol-related presentations to Australasian emergency departments. Med 7 Aust 2014;201:584-7.

16. Brennan PL. Functioning and health service use among elderly nursing home residents with alcohol use disorders: findings from the national nursing home survey. Am 7 Geriatr Psychiatry 2005;13:475-83.

17. Willmore J, Marko T, Taing D, et al. The burden of alcohol-related morbidity and mortality in Ottawa, Canada. PLoS One 2017;12:e0185457.

18. Cherpitel CJ. Drinking patterns and problems, drug use and health services utilization: a comparison of two regions in the US general population. Drug Alcohol Depend 1999;53:231-7.

19. Roos LL, Gupta S, Soodeen R, et al. Data quality in an information-rich environment: Canada as an example. Can f Aging 2005;24(Suppl 1):153-70.

20. Roos LL, Nicol JP. A research registry: uses, development, and accuracy. 7 Clin Epidemiol 1999;52:39-47.

21. Mustard CA, Derksen S, Berthelot JM, et al. Assessing ecologic proxies for household income: a comparison of household and neighbourhood level income measures in the study of population health status. Health Place 1999;5:157-71. 
22. Weiner JP, editor. The Fohns Hopkins ACG Case-Mix System documentation \& application manual, version 10. Baltimore: Johns Hopkins University; 2001.

23. Myran DT, Hsu AT, Smith G, et al. Rates of emergency department visits attributable to alcohol use in Ontario from 2003 to 2016: a retrospective population-level study. CMA7 2019;191:E804-10.

24. Canadian tobacco alcohol and drugs (CTADS): 2015 summary. Ottawa: Government of Canada; 2017. Available: https://www.canada.ca/en/health-canada/services/ canadian-tobacco-alcohol-drugs-survey/2015-summary.html (accessed 2017 Oct. 29).

25. Zelner I, Koren G. Alcohol consumption among women. 7 Popul Ther Clin Pharmacol 2013;20:e201-6.

26. Devries KM, Child J, Bacchus L, et al. Intimate partner violence victimization and alcohol consumption in women: a systematic review and meta-analysis. Addiction 2014;109:379-91.

27. Women and alcobol. Ottawa: Canadian Centre on Substance Abuse; 2014. Available: https://www.ccsa.ca/sites/default/files/2019-05/CCSA-Women -and-Alcohol-Summary-2014-en.pdf (accessed 2020 Oct. 10).

28. Oleski J, Mota N, Cox B, et al. Perceived need for care, help seeking, and perceived barriers to care for alcohol use disorders in a national sample. Psychiatr Serv 2010;61:1223-31.

29. Miquel L, Gual A, Vela E, et al. Alcohol consumption and inpatient health service utilization in a cohort of patients with alcohol dependence after 20 years of follow-up. Alcohol Alcobol 2017;52:227-33.

30. Grant BF, Goldstein RB, Saha TD, et al. Epidemiology of DSM-5 alcohol use disorder: results from the national epidemiologic survey on alcohol and related conditions III. 7AMA Psychiatry 2015;72:757-66.

31. Wray TB, Celio M, Kahler C, et al. Daily co-occurence of alcohol use and high-risk sexual behavior among heterosexual, heavy drinking emergency department patients. Drug Alcohol Depend 2015;152:109-15.

32. Lejuex CW, Magidson J, Mitchell S, et al. Behavioral and biological indicators of impulsivity in the development of alcohol use, problems, and disorders. Alcobol Clin Exp Res 2010;34:1334-45.

33. Canada's low-risk alcohol drinking guidelines. Ottawa: Canadian Centre on Substance Use and Addiction; 2017. Available: www.ccsa.ca/Resource Library/2012-Canada-Low-Risk-Alcohol-Drinking-Guidelines-Brochure-en. pdf (accessed 2020 Oct. 10)

34. O'Donnell A, Anderson P, Newbury-Birch D, et al. The impact of brief alcohol interventions in primary healthcare: a systematic review of reviews. Alcobol Alcobol 2014;49:66-78.

35. Bertholet N, Daeppen J, Wietlisbach V, et al. Reduction of alcohol consumption by brief alcohol intervention in primary care: systematic review and meta-analysis. Arch Intern Med 2005;165:986-95.

36. Spithoff S, Kahan M. Primary care management of alcohol use disorder and at-risk drinking - part 2: counsel, prescribe, connect. Can Fam Pbysician 2015;61:515-21.

37. Kim HM, Smith E, Stano C, et al. Validation of key behaviourally based mental health diagnoses in administrative data: suicide attempt, alcohol abuse, illicit drug abuse and tobacco use. BMC Health Serv Res 2012;12:18.

38. Probst C, Manthey J, Martinez A, et al. Alcohol use disorder severity and reported reasons not to seek treatment: a cross-sectional study in European primary care practices. Subst Abuse Treat Prev Policy 2015;10:32.
Affiliations: Department of Psychiatry (Bolton, Leong, Konrad, Nepon), Manitoba Centre for Health Policy (Bolton, Ekuma, Prior, Enns, Singal, Paillé, Finlayson, Nickel), College of Pharmacy (Leong) and Department of Community Health Sciences (Nickel), Rady Faculty of Health Sciences, University of Manitoba, Winnipeg, Man.

Contributors: James Bolton and Nathan Nickel conceptualized the study, led the study design and oversaw data acquisition. Okechukwu Ekuma and Heather Prior conducted the statistical analyses. All of the authors were involved in interpreting the study findings. James Bolton, Christine Leong and Nathan Nickel drafted the manuscript, and all of the authors revised the manuscript for important intellectual content, approved the final version for publication and agreed to be accountable for the work.

Funding: Funding for this work was provided as part of a contract between the University of Manitoba and the Manitoba Government's Ministry of Health, Seniors and Active Living for a study led by Nathan Nickel. The results and conclusions are those of the authors, and no official endorsement by Manitoba Health, Seniors and Active Living is intended or should be inferred. James Bolton is supported by a Canadian Institutes of Health Research operating grant and a Brain and Behaviour Research Foundation Young Investigator Award.

Data sharing: The data used in this study were derived from administrative health and social data as a secondary use. The data were provided under specific data-sharing agreements only for approved use at the Manitoba Centre for Health Policy (MCHP). The original source data are not owned by the researchers or by MCHP and as such cannot be provided to a public repository. The original data source and approval for use have been noted in the acknowledgments section that follows. Where necessary, source data specific to this article or project may be reviewed at MCHP with the consent of the original data providers, along with that of the required privacy and ethics review bodies.

Acknowledgements: The authors thank Dan Chateau for sharing his biostatistical expertise for this study and acknowledge the Manitoba Centre for Health Policy (MCHP) for use of data contained in the Population Health Research Data Repository (HIPC project no. 2014/2015-30). The results and conclusions are those of the authors, and no official endorsement by the MCHP, Manitoba Health or other data providers is intended or should be inferred. This analysis made use of the Johns Hopkins Resource Utilization Bands, based on the Johns Hopkins Adjusted Clinical Group Case-Mix System, version 10 (cited as reference 22).

Supplemental information: For reviewer comments and the original submission of this manuscript, please see www.cmajopen.ca/content/8/4/ E762/suppl/DC1. 